\title{
Modular EUV Source for the Next Generation Lithography
}

\author{
Olivier Sublemontier ${ }^{* 1}$, Marylène Rosset-Kos ${ }^{* 1}$, Tiberio Ceccotti ${ }^{* 1}$, Jean-François Hergott ${ }^{* 1}$, \\ Thierry Auguste ${ }^{* 1}$, Didier Normand ${ }^{* 1}$, Martin Schmidt ${ }^{* 1}$, François Beaumont ${ }^{* 2}$, Daniel Farcage ${ }^{* 2}$, \\ Guy Cheymol ${ }^{* 2}$, Jean-Marc Le Caro ${ }^{* 2}$, Philippe Cormont ${ }^{* 2}$, Patrick Mauchien ${ }^{* 2}$, Pierre-Yves Thro ${ }^{* 2}$, \\ Jacky Skrzypczak $^{* 3}$, Sophie Muller ${ }^{* 3}$, Emanuel Marquis ${ }^{* 3}$, Benoît Barthod ${ }^{* 4}$, \\ Isabelle Gaurand $^{* 4}$, Magali Davenet ${ }^{* 4}$ and Roland Bernard ${ }^{* 4}$ \\ ${ }^{* 1}$ Commissariat à l'Energie Atomique, DSM/IRAMIS/SPAM, 91191 Gif sur Yvette, France \\ E-mail: Olivier.sublemontier@cea.fr \\ ${ }^{* 2}$ Commissariat à l'Energie Atomique, DEN/DPC/SCP, 91191 Gif sur Yvette, France \\ *3 Thales Laser, Domaine de Corbeville, Route départementale 128, BP 46, 91401 Orsay, France \\ ${ }^{*}$ Alcatel Vacuum Technology France, 98 Avenue de Brogny, 74009 Annecy, France
}

The present work, performed in the frame of the EXULITE project, was dedicated to the design and characterization of a laser-plasma-produced extreme ultraviolet (EUV) source prototype at 13.5 $\mathrm{nm}$ for the next generation lithography. It was conducted in cooperation with two laboratories from CEA, ALCATEL and THALES. One of our approach originalities was the laser scheme modularity. Six Nd:YAG laser beams were focused at the same time on a xenon filament jet to generate the EUV emitting plasma. Multiplexing has important industrial advantages and led to interesting source performances in terms of in-band power, stability and angular emission properties with the filament jet target. A maximum conversion efficiency (CE) value of $0.44 \%$ in $2 \pi$ sr and $2 \%$ bandwidth was measured, which corresponds to a maximum in band EUV mean power of $7.7 \mathrm{~W}$ at a repetition rate of $6 \mathrm{kHz}$. The EUV emission was found to be stable and isotropic in these conditions. DOI:10.2961/jlmn.2011.02.0004

Keywords: EUV lithography, EUV source, laser multiplexing, xenon filament jet, laser-produced plasma

\section{Introduction}

The extreme ultraviolet (EUV) lithography technology at $13.5 \mathrm{~nm}$ wavelength and within $2 \%$ bandwidth is the leading candidate for use in the next generation highvolume manufacturing (HVM) of integrated circuits with features smaller than $22 \mathrm{~nm}$. The development of a high power and reliable EUV source remain a critical issue to enable the pilot line introduction as early as 2013 [1-3]. This subject has motivated an intense research effort worldwide during the last decade both theoretically $[4,5]$ and experimentally $[6,7]$.

As the gas discharge plasma (GDP) technology was already chosen for integration on alpha and beta-tools [8], the competition is still open for HVM with the laser-produced plasma (LPP) technology EUV sources [9] and the new concept of hybrid laser-induced discharge [10]. Several target materials and phases were investigated for LPP sources. One of the first of them with promising properties was the xenon filament jet $[11,12]$. This choice was mainly driven by the very low contamination contribution from this material unlike metallic competitor targets.

The present work relates on an original modular scheme for the LPP EUV source developed in the frame of the Exulite consortium project. Six Nd:YAG laser beams at $1.06 \mu \mathrm{m}$ wavelength were focused at the same time on a xenon filament target to generate the EUV emitting plasma. In addition to the modularity aspects, we demonstrate that laser multiplexing shows interesting properties in terms of efficiency, stability and angular emission.

\section{Setup}

The source overall scheme is presented in Fig. 1. Six laser beams from identical modules were routed and focused separately in a high vacuum interaction chamber. The vacuum system, provided by ALCATEL, was composed of two $2300 \mathrm{l} / \mathrm{min}$ turbo pumps and a $500 \mathrm{~m}^{3} / \mathrm{h}$ roots pump. Each beam was focused on the xenon filament jet at the same time.

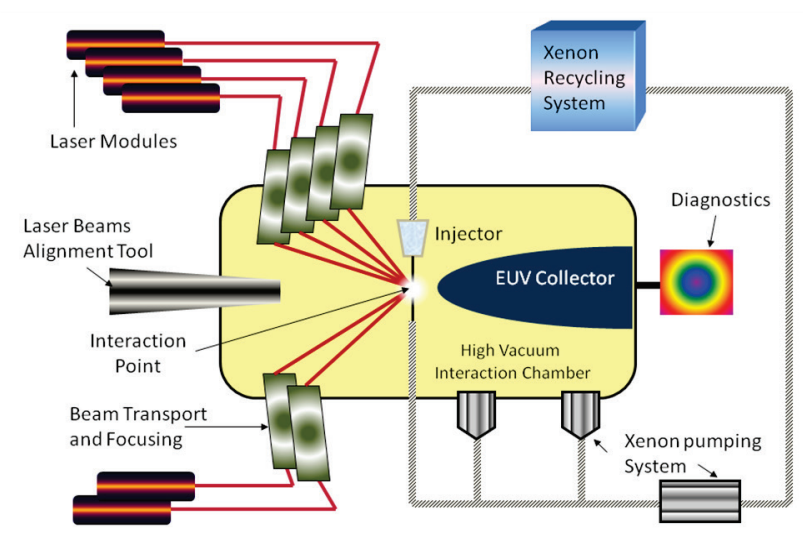

Fig. 1 The spider-like attack EUV source scheme. Six Nd:YAG laser beams are focused at the same time on a xenon filament jet.

The xenon pumping system exhaust was connected to a cryogenic xenon recycling setup. The recycling could oper- 
ate $24 \mathrm{~h}$ a day if needed and the efficiency was close to $100 \%$. The collector chamber was removable and was equipped with an independent pumping system. The diagnostic set included a synchrotron calibrated in-band absolute radiation measurement tool at $13.5 \mathrm{~nm}$, large band EUV calibrated photodiodes, a EUV spectrometer and a debris analyzer.

The use of several laser modules focused at the same time on the same point led to a simpler system with enhanced redundancy. This crucial advantage concerns the construction of the system, but also its use in an industrial environment since it is possible for the EUV source to continue to operate in case of a laser module failure or maintenance.

\subsection{Multiple-beam focusing}

The multiple-beam focusing was designed in such a way to optimize EUV radiation collection from the plasma. The setup maximum capacity is 10 laser modules and the design is scalable to extend up to 20 modules. In the present work, 6 laser modules were used simultaneously. The multiple-beam focusing scheme is presented in Fig. 2. Fig. 2 (a) is a $2 \mathrm{D}$ presentation of the angular space management in the transverse plane. Fig. 2(b) is a 3D presentation including the laser beams, the focusing mirrors and the 3 pieces collecting device. A picture of a focusing mirror is given.
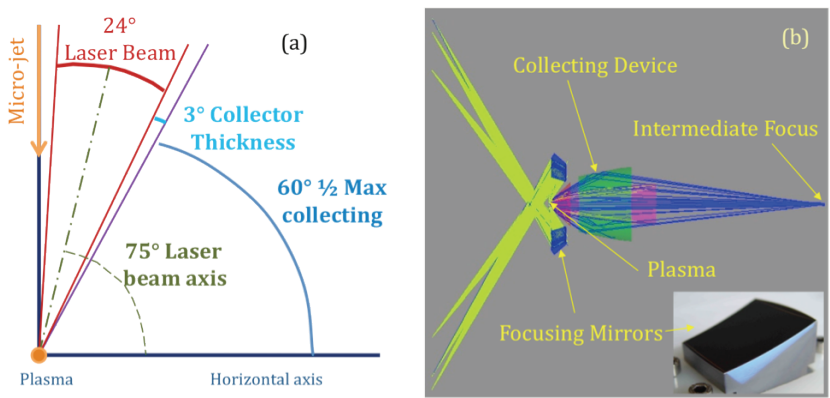

Fig. 2 Multiple-beam focusing design. Angular space management (a) and 3D laser beams focalization and EUV collection.

The maximum EUV collection angle from the horizontal axis was $60^{\circ}$. This allowed a theoretical transmission efficiency of $27 \%$ for a 3 parts elliptical grazing incidence collecting device, taking into account the optical reflectivity of the elements at $13.5 \mathrm{~nm}$. The collector thickness was responsible for additional $3^{\circ}$. The laser beam axes were disposed at $75^{\circ}$ from the horizontal axis in a cylindrical symmetry from this axis. This allowed a high numerical aperture for the laser beams, which was needed for a relatively small focusing spot. The spot diameter was $50 \mu \mathrm{m}$ $\left(1 / \mathrm{e}^{2}\right)$ at the focal point. The pre-alignment of all the focused laser beams were performed on a $50 \mu \mathrm{m}$ glass sphere positioned in place of the interaction point and with the laser beams attenuated by a factor of $10^{6}$. Angles between the different laser beam axes were $22^{\circ}$. Mirrors were high quality focusing units with low deformation response to thermal load. The mirror support was water cooled. No shift of the focused beams was observed during the full power source runs. For financial reasons, we were not able to finalize the collecting device construction as the conception work was complete.

\subsection{Laser modules}

The modules were diode-pumped solid-state lasers in with Nd:YAG rods pumped by cw high power diode bars. The cw pumping was chosen for high efficiency with high repetition rate $(>3 \mathrm{kHz})$ operation. Moreover, the lifetime of cw diodes may reach up to 20.000 hours. For a better optical-to-electrical efficiency, we chose to develop a power-oscillator-power-amplifier (POPA) configuration instead of the standard master-oscillator-power-amplifier (MOPA) system. There was then no need for pre-amplifiers because of the efficient extraction of the energy stored in the first amplifier by the output from the oscillator. The beam quality was a compromise between efficiency and focus ability. A $\mathrm{M}^{2}$ between 10 and 15 was obtained and maintained through the amplification chain. The power oscillator, presented in Fig. 3(a), was specifically developed at CEASaclay and a commercial version presented in Fig. 3(b) is now available at THALES.

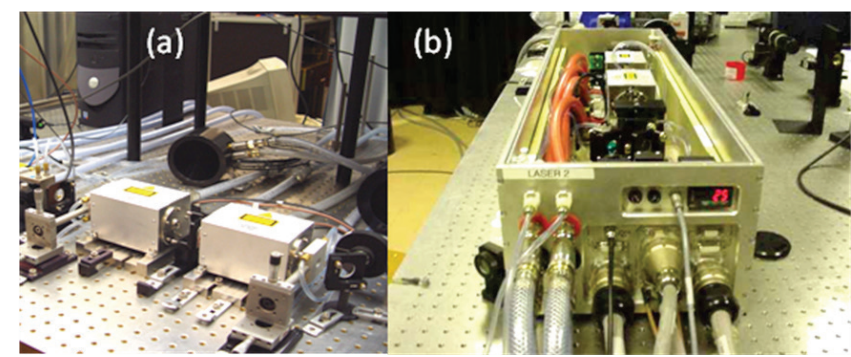

Fig. 3 The laser modules at the Development stage at CEA (a) and the corresponding commercial version from THALES (b).

The output average power was $480 \mathrm{~W}$ at $10 \mathrm{kHz}$ repetition rate. The pulse duration was $30 \mathrm{~ns}$ at $6 \mathrm{kHz}$, and around $40 \mathrm{~ns}$ at $10 \mathrm{kHz}$. This led to an intensity of 7.5.10 ${ }^{10} \mathrm{~W} / \mathrm{cm}^{2}$ on a $50 \mu \mathrm{m}$ diameter spot with one module. The optical-to-optical and optical-to-electrical efficiencies of the laser were respectively $21 \%$ and $7 \%$. The time jitter was $1.2 \mathrm{~ns}$ rms and the power fluctuations shot to shot were less than 3\%. Preliminary experiments were carried out by adding an identical amplifier stage to the oscillator. $860 \mathrm{~W}$ power were obtained with a base of a $440 \mathrm{~W}$ oscillator and $\mathrm{M}^{2}$ was slightly degraded from 13 to 16 . It is possible to produce 1.5 to $2 \mathrm{~kW}$ modules allowing up to $100 \mathrm{~W}$ EUV power source.

\subsection{Stability and EUV doze control}

The modular multiplexed LPP source requires an excellent spatial stability of the laser beams on target. To this end, a close-loop feedback control was designed for each laser beam to compensate short and long-term fluctuations. This was achieved by tracking the near field and the far field of a spatially optimized laser beam in order to maintain its position. 
In addition, an intelligent doze control system was designed. The principle was based on the temporal control of one of the multiplexed lasers. Assuming that the number of shots needed for the illumination of a wafer area element was 50, the EUV doze effectively received for the first 49 was measured. The doze required for the last shot was then deduced and adjusted by controlling the temporal shift of one of the lasers with the help of an algorithm which takes into account the continuous running window. The temporal shift was easily and rapidly realized between two shots.

\subsection{Xenon filament jet}

The xenon microfilament jet was produced by a commercial system provided by Microliquids $\mathrm{GmbH}$. The gaseous xenon was cooled down between $-130^{\circ} \mathrm{C}$ to $-110^{\circ} \mathrm{C}$ temperature range before the nozzle entrance by a specific cryostat. Pressures from 10 to 30 bars were used to obtain a stable $35 \mu \mathrm{m}$ diameter xenon jet. A picture of the filament is shown in Fig. 4(a). The quartz nozzle can be seen on the top of the picture. The jet appeared like a filament which then entered into an inverse differential pumping system to keep a sufficiently high vacuum in the interaction chamber. Fig. 4(b) shows the same filament jet irradiated by the 6 laser beams focused on it. The distance between the nozzle and the focusing point was $12 \mathrm{~mm}$. This was a compromise between stability of the jet and degradation of the nozzle from ionic debris emitted by the plasma.
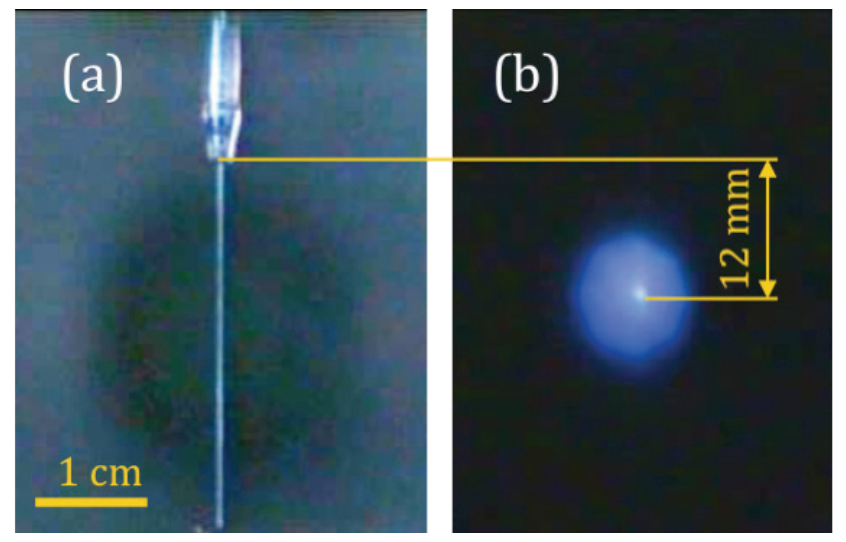

Fig. 4 The xenon filament jet (a) and the same target irradiated by the six laser beams (b).

The filament stability was carried out from high magnification laser shadowscopy measurements. The results are presented in Fig. 5. From the bottom up, the jet position, the jet diameter and the density variations of the jet are presented. As the jet density and diameter variations were almost negligible, the jet position is changing rapidly by $+/-5 \mu \mathrm{m}$ within few minutes. Important experimental efforts were engaged to find the appropriate conditions of pressure and temperature for each nozzle to have these position variations acceptable (less than $+/-5 \mu \mathrm{m}$ ) for suitable interaction conditions. The nozzle was mounted on xy micro translation stages with $1 \mu \mathrm{m}$ positioning precision. The alignment procedure will be discussed later.

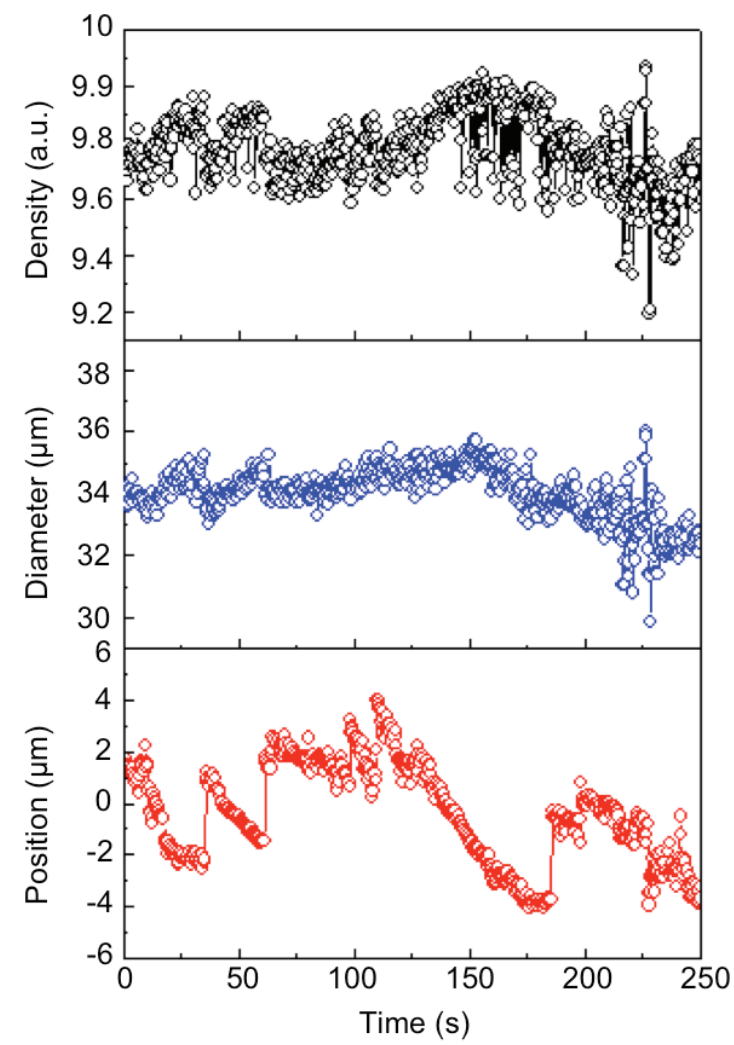

Fig. 5 Xenon filament jet stability measurements. From the bottom up: position, diameter and density variations.

\section{Multi-beam alignment procedure}

After pre-alignment of each laser beam focus on a spherical $50 \mu \mathrm{m}$ ball at atmospheric pressure, the target position was adjusted under vacuum in order to get the xenon filament target as close as possible to the crossing laser beams. All the laser beams were then attenuated by a factor of $10^{6}$.

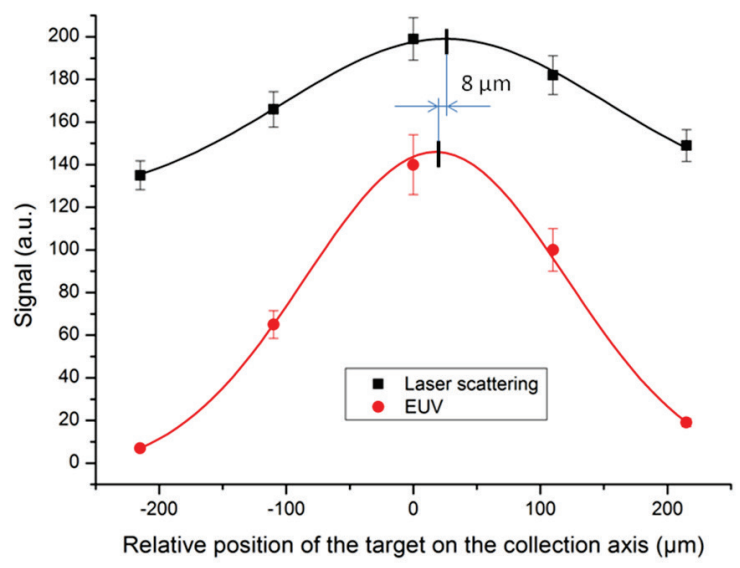

Fig. 6 EUV (round dots) and diffusion (square dots) signals as a function of the target lateral position. The positions of both optima are as close as $8 \mu \mathrm{m}$ from each other. 
A homemade laser alignment tool composed of a high sensitive camera equipped with high magnification optics measured the interaction of the lasers with the target. The laser scattered signal from the interaction with only one laser beam was efficiently detected. That way, laser beams were precisely aligned one by one on the xenon filament jet. Using this method, we estimate the uncertainty on the laser beam positions at about $+/-8 \mu \mathrm{m}$. Fig. 6 shows a comparison between laser scattered signal with attenuated lasers and in-band EUV photons from the source with the full laser power as a function of the transversal position of the target. Both signals were fitted by a Gaussian. The maxima are only $8 \mu \mathrm{m}$ from each other, which is below the measurement and Gaussian fit uncertainties. The alignment method was then validated. This procedure has been very useful for preserving the nozzle lifetime.

\section{Results and discussion}

The six laser beams were "spider-like" focused on the xenon filament jet resulting in a total power of $1752 \mathrm{~W}$ on target. The filament diameter was $35 \mu \mathrm{m}$ and the focus diameters of the laser beams were $50 \mu \mathrm{m}$. The repetition rates were $6 \mathrm{kHz}$ and $10 \mathrm{kHz}$ and the pulse durations were $30 \mathrm{~ns}$ and $40 \mathrm{~ns}$ respectively, with a jitter of less than $1.2 \mathrm{~ns}$ in both cases. The distance from the nozzle to the interaction point was $12 \mathrm{~mm}$. The absolute EUV in-band emitted power was measured with a synchrotron calibrated EUV inband power meter, placed in place of the EUV collector chamber. This system consisted of a diaphragm, a MoSi multilayer EUV mirror optimized for $13.5 \mathrm{~nm}$ wavelength and with a known bandwidth, and a EUV photodiode from IRD (International Radiation Detectors) with a deposited $100 \mathrm{~nm}$ zirconium filter. The measurement scheme is presented in Fig. 7. Fig. 7(a) is a side view from the in-band power meter and Fig. 7(b) is a top view. L1, L2, L3, L4, L5 and $\mathrm{L} 6$ are the laser beams.

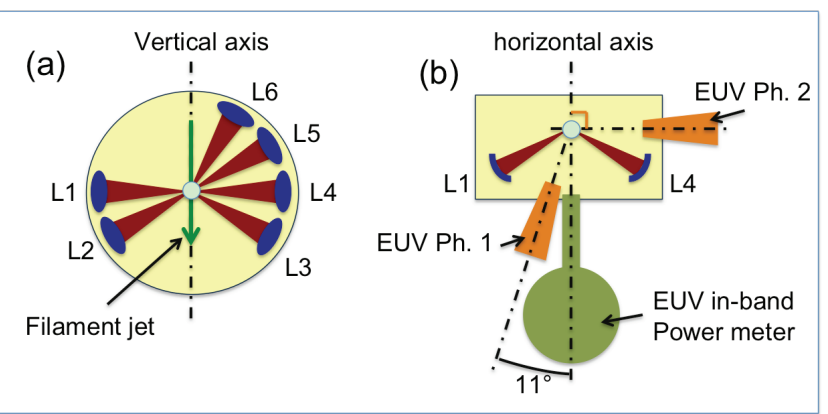

Fig. 7 EUV measurement management with a side view from the in-band power meter (a) and a top view (b).

In addition to the in-band power meter, two identical calibrated EUV photodiodes with a deposited zirconium film were used to probe the isotropic properties of the source. Their bandwidths were approximately $8 \mathrm{~nm}$ centered on $13.5 \mathrm{~nm}$ in wavelength. The first one, EUV Ph. 1, was placed as close as possible to the in-band power meter. The angle between the horizontal axis and EUV $\mathrm{Ph}$. 1 was $11^{\circ}$. The second one was placed orthogonally to the hori- zontal axis. The distances and solid angles from the plasma were identical for both EUV photodiodes. All signals were processed in the same time by a high-speed multichannel digital calibrated oscilloscope. The EUV signals received at the same time by both photodiodes using the full laser power at $10 \mathrm{kHz}$ repetition rate are plotted in Fig. 8 . Fig. 8(a) is for EUV Ph. 1 and Fig. 8(b) is for EUV Ph. 2. 20 pulses from consecutive laser shots were taken. In both cases, the EUV signals were constant and were approximately the same for both photodiodes. This suggests that the EUV emission was isotropic at least in the half-space containing the in-band power meter. We believe this is another advantage of the spider like attack in comparison to the single-laser beam one. This allows us to announce a real average EUV power of our source in $2 \pi$ sr and $2 \%$ bandwidth. The EUV Ph. 2 was moved to the symmetrical place from the horizontal axis and the results were comparable. The isotropic properties were also verified with a repetition rate of $6 \mathrm{kHz}$.

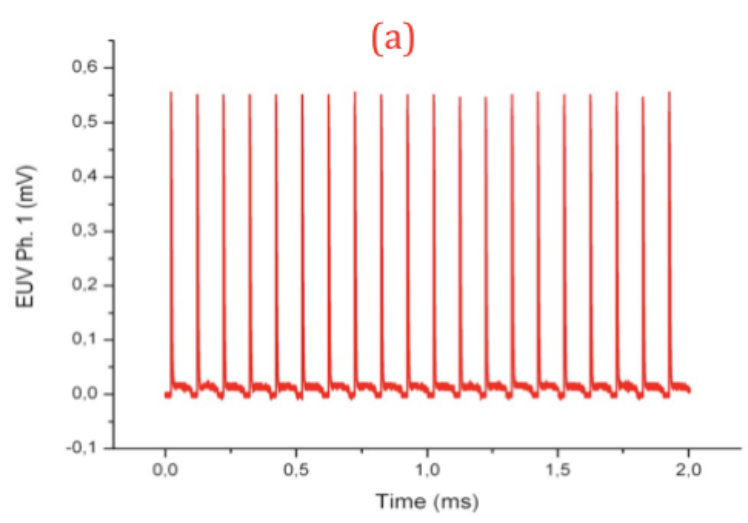

(b)

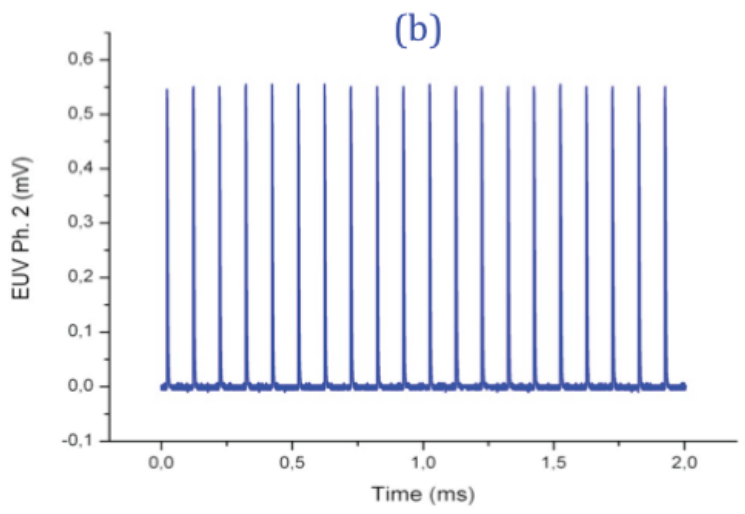

Fig. 8 EUV signal received simultaneously by both photodiodes EUV Ph. 1 (a) and EUV Ph. 2 (b) within 20 full power laser shots.

The stability of the source was checked in different laser configurations. In each condition, the conversion efficiency (CE) was deduced from the absolute in-band power measurements at $13.5 \mathrm{~nm}$ for 20 consecutive laser shots. Fig. 9 shows the EUV in-band energy per pulse delivered at $13.5 \mathrm{~nm}$ in $2 \pi \mathrm{sr}$ with two of these configurations. Blue squares correspond to the six laser beams full power with a $6 \mathrm{kHz}$ repetition rate. Red triangles correspond to the half of the full laser power with only L1, L4 and L5. 
The maximum $\mathrm{CE}$ obtained at full laser power $(1752 \mathrm{~W})$ on target is $0.44 \%$. To our knowledge, this is the best result obtained with a xenon target without any prepulse and with pulse duration as high as $30 \mathrm{~ns}$. This corresponds to a maximum EUV power emitted by the source point of $7.7 \mathrm{~W}$ at $13.5 \mathrm{~nm}$, in $2 \%$ bandwidth and $2 \pi$ sr. The average value of the $\mathrm{CE}$ is $0.41 \%$, very close to the maximum with 6 lasers. The source stability is typically $3.3 \%$ in $1 \sigma$ for 20 shots without the EUV doze control system. This stability was observed during about 12 minutes, until the quartz nozzle of the filament jet was irreversibly damaged by ionic debris from the plasma. In comparison, with only 3 laser beams, the maximum $\mathrm{CE}$ was quite high, around $0.37 \%$, but the average $\mathrm{CE}$ was as small as $0.07 \%$. The latter configuration demonstrates the instability of the source in these conditions, which is typically around $63 \%$ in $1 \sigma$ for 20 shots.

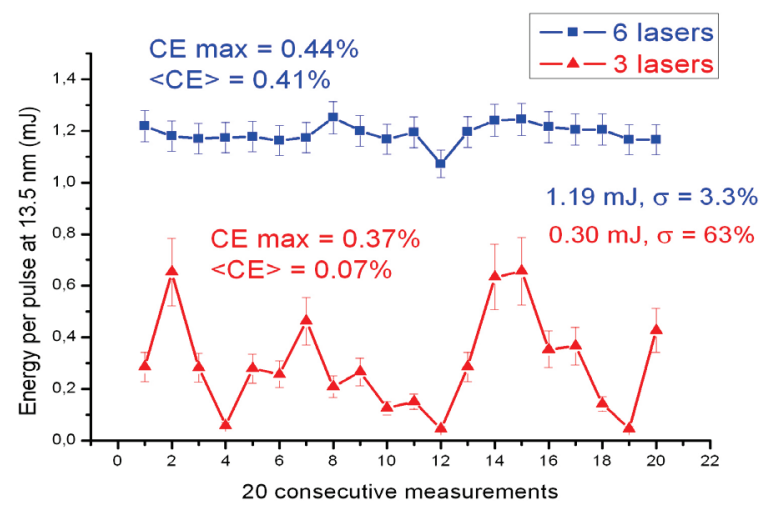

Fig. 9 Energy per pulse for 20 consecutive laser shots with six laser beams (squares) and three laser beams (triangles).

Two hypotheses were considered to explain the differences between 3 and 6 laser beams. The first one considered that there was a kind of threshold effect with 3 laser beams that caused the instabilities while keeping the possibility to have some pulses with quite high CE. The second one considered that the laser beam multiplexing resulted in better interaction shot to shot stability and efficiency. As the maximum $\mathrm{CE}$ with 3 laser beams was close to the maximum CE with 6 laser beams, a threshold effect could not be the only reason for a so high instability. The instability of the source in the 3-laser beams configuration could be due to a high sensitivity of the EUV emission properties, including isotropy, to small position variations of the target or the laser beams. This sensitivity could be dramatically reduced by increasing the number of lasers because of the independence and randomness of small laser position variations. That way, if target or laser beam position variations occurred, one of the other laser beams hit the target and the very fast-expending plasma interacted early with the other laser beams, when the plasma density was still high, resulting in smoothing EUV emission fluctuations.

In addition, Sizyuk and coworkers [5] suggested that multiple laser beam on a single target could lead to an increase of the EUV emission efficiency in comparison to single-beam one with equivalent irradiation energy. This theoretical work took into account plasma heat conduction and hydrodynamic processes. The relatively high maximum CE observed with three lasers in our experiments seems to be in agreement with theses simulation results. At first, this might seem surprising, as multiple-beam focusing with very different angles on a cylindrical or a spherical target is unfavorable from the pure laser intensity point of view. Yet the EUV radiation in this case is the result of matching the three lasers together, as at least three lasers are needed to extract EUV radiation from the plasma. The relatively high maximum $\mathrm{CE}$ observed in the three-laser beam configuration may be assigned to an efficient but unstable plasma process generated by the combination of the three lasers. The EUV emission was slightly more efficient and much more stable as the number of lasers was increased up to six. Therefore, we assume that the EUV emitted radiation was then less sensitive to small shot-toshot laser beam position variations, resulting in a more stable emission.

\section{Conclusion}

A modular LPP EUV source prototype at $13,5 \mathrm{~nm}$ was designed for the next generation lithography. The multiplexing of six power laser beams on a xenon filament jet target led to a maximum conversion efficiency of $0,44 \%$, corresponding to an average power of 7,7 W at $13,5 \mathrm{~nm}$ in $2 \pi$ sr and $2 \%$ bandwidth. The "spider - like" laser architecture, beyond the obvious but important advantages arising from redundancy, shows interesting properties in terms of efficiency, isotropy and stability. The relatively high $\mathrm{CE}$ reported here with a xenon target is in agreement with previous theoretical work involving multiple laser beam irradiation scheme.

\section{References}

[1] H.J. Levinson: J. Micro-Nanolitho. MEMS MOEMS, 8, (2009) 041501

[2] J. Benshop, V. Banine, S. Lok and E. Loopstra: J. Vac. Sci. Technol. B, 26, (2008) 2204

[3] S. Wurm: J. Photopolymer Sci. Technol., 22, (2009) 31

[4] T. Auguste, F. De Gaufridy De Dortan, T. Ceccotti, J.F. Hergott, O. Sublemontier, D. Descamps and M. Schmidt: J. Appl. Phys., 101, (2007) 043302

[5] V. Sizyuk, A. Hassanein and T. Sizyuk: J. Appl. Phys., 100, (2006) 103106

[6] G. Cheymol, P. Cormont, P-Y. Thro, O. Sublemontier, M. Schmidt, B. Barthod: Patent WO06000718 (2006)

[7] U. Stamm, M. Yoshioda, J. Kleinschmidt, C. Ziener, G. Schriever, M.C. Schurmann, G. Hergenam and V.M. Borisov: Emerg. Litho. Technol. XI, PTS 1 and 2, (2007) 6517

[8] H. Meiling, E. Boon, N. Buzing, K. Cummings, O. Frijns, J. Galloway, M. Goethals, N. Harned, B. Hultermans, R. de Jonge, B. Kessels, P. Kurtz, S. Lok, M. Lowisch, J. Mallman, B. Pierson, K. Ronse, J. Ryan, E. Smitt-Weaver, M. Tittnich, C. Wagner, A. van Dijk and J. Zimmerman: Emerg. Lotho. Technol. XII, PTS 1 and 2, (2008) 6921 
[9] N.R. Bowering, I.V. Fomenkov, D.C. Brandt, A.N. Bykanof, A.I. Ershov, W.N. Partlo, D.W. Myers, N.R. Farrar, G.O. Vaschenko, O.V. Khodykin, J.R. Hoffman, C.P. Chrobak, S.N. Srivastava, I. Ahmad, C. Rajyaguru, D. Golich, D.A. Vidusek, S. De Dea and R.R. Hou: J. Micro-Nanolitho. MEMS MOEMS, 8, (2009) 041504

[10] V.M. Borisov, A.Y. Vinokhodov, A.S. Ivanov, Y.B. Kiryukhin, V.A. Mishchenko, A.V. Prokof'ev and O.B. Khristoforov: Quantum Electron., 39, (2009) 967
[11] B. A. M. Hansson, L. Rymell, M. Berglund, O. Hemberg, E. Janin, J. Thoresen, S. Mosesson, J. Wallin and H. M. Hertz: Emerg. Litho. Technol. VI, Proc. SPIE, 4688, (2002) 102

[12] Y. Ueno, T. Ariga, G. Soumagne, T. Higashiguchi, S. Kubodera, I. Pogorelsky, I. Pavlishin, D. Stolyarov, M. Babzien, K. Kushe and V. Yakimenko: Appl. Phys. Lett., 90 (2007) 191503

(Received: October 06, 2010, Accepted: May 12, 2011) 